\title{
PROPERTIES OF THE RESOLVENT OF THE LAPLACE OPERATOR ON A TWO-DIMENSIONAL SPHERE AND A TRACE FORMULA
}

\section{A.I. ATNAGULOV, V.A. SADOVNICHY, Z.YU. FAZULLIN}

\begin{abstract}
In the work we study the properties of the resolvent of the Laplace-Beltrami operator on a two-dimensional sphere $S^{2}$. We obtain the regularized trace formula for the Laplace-Beltrami operator perturbed by the operator of multiplication by a function in $W_{2}^{1}\left(S^{2}\right)$.
\end{abstract}

Keywords: resolvent, kernel, Laplace-Beltrami operator, perturbed operator.

Mathematics Subject Classification: 47B10, 47B15, 47A55

\section{INTRODUCTION}

Let

$$
H_{0}=-\frac{1}{\sin \theta} \frac{\partial}{\partial \theta} \sin \theta \frac{\partial}{\partial \theta}-\frac{1}{\sin ^{2} \theta} \frac{\partial^{2}}{\partial \varphi^{2}}
$$

be the Laplace-Beltrami operator on a two-dimensional sphere $S^{2}$. The work is devoted to the studying the spectral properties of a perturbation of this operator $H u=H_{0} u+V u$. Namely, it is devoted to proving the Gel'fand-Levitan formula for the regularized trace of the LaplaceBeltrami operator perturbed by the operator $V$ of the multiplication by a function $\nu(\omega), \omega \in S^{2}$. The trace of the Laplace-Beltrami operator perturbed by an odd function $\nu(\omega) \in C^{\infty}\left(S^{2}\right)$ was obtained first in 1993-1996 in the works [1], [2] (although the problem was proposed by I.M. Gel'fand in 1962). We note that the conditions of the oddness and belonging to the class $C^{\infty}\left(S^{2}\right)$ for the function $\nu(\omega)$ were essential for the method employed in these works. The next step in this problem was made in works [3]- [5] based on a way of summing the second correctors of the perturbation theory. In these works, the classic Gel'fand-Levitan formula was obtained for arbitrary function (not necessarily odd) $\nu(\omega)$ of a finite smoothness, and in the work [5] the only condition assumed was $\nu(\omega) \in C^{2}\left(S^{2}\right)$.

As it is turned out, for further weakening of the conditions for the perturbation $\nu(\omega)$ one needed a more detailed study of the properties of the kernel $R_{0}\left(\omega, \omega_{0}, \lambda\right)$ of the resolvent for the Laplace-Beltrami operator (Theorem 1), of the kernel $R_{0 n}\left(\omega, \omega_{0}, \lambda\right)$ of the reduced resolvent(Theorem 22). On the base of these studies and the approach of the work [5], the trace formula for the Laplace-Beltrami operator was obtained for the perturbations $\nu(\omega)$ in the class $W_{2}^{1}\left(S^{2}\right)$.

We note that Sections 2 and 3 are devoted to the detailed exposition of the results of the work [6].

A.I. Atnagulov, V.A. Sadovnichy, Z.Yu. Fazullin, Properties of the Resolvent of the LAPLACE OPERATOR ON A TWO-DIMENSIONAL SPHERE AND A TRACE FORMULA.

(C) Atnagulov A.I., Sadovnichy V.A., Fazullin Z.Yu. 2016.

The work is supported by the grant no. 01201456408 of the Ministery of Education and Science of Russia.

Submitted June 26, 2016. 


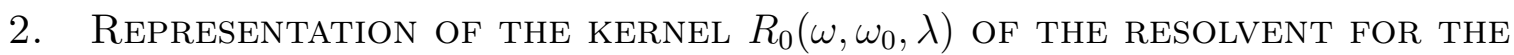 LAPLACE-BELTRAMI OPERATOR}

It is well-known [7] that the kernel $R_{0}\left(\omega, \omega_{0}, \lambda\right)$ of the resolvent $R_{0}(\lambda)=\left(H_{0}-\lambda\right)^{-1}$ for the operator $H_{0}$ (Laplace-Beltrami operator) in $L^{2}\left(S^{2}\right)$ is equal to

$$
R_{0}\left(\omega, \omega_{0}, \lambda\right)=\frac{1}{4 \pi} \sum_{n=0}^{\infty} \frac{(2 n+1) P_{n}(\cos \alpha)}{n(n+1)-\lambda}
$$

where $\alpha$ is the angle between vectors $\omega, \omega_{0} \in S^{2}, P_{n}(\cos \alpha)$ is a Legendre polynomial, and

$$
P_{n}\left(\omega, \omega_{0}\right)=\frac{2 n+1}{4 \pi} P_{n}(\cos \alpha)
$$

is the kernel of the orthogonal projector $P_{n}$ projecting on the eigenspace associated with the eigenvalue $\lambda_{n}=n(n+1)$ of the operator $H_{0}$ and the multiplicity of $\lambda_{n}$ is equal to $(2 n+1)$.

On the other hand, it is known (see, for instance, [8, Sect. 4.3, 4.5]) that the sequence

$$
f_{n}(\alpha)=\sqrt{n+1 / 2} \sqrt{\sin \alpha} P_{n}(\cos \alpha), n=0,1, \ldots,
$$

is an orthonormal basis formed by the eigenfunctions for the Dirichlet problem for the ordinary differential operator

$$
M f(\alpha)=-f^{\prime \prime}(\alpha)-\left(4 \sin ^{2} \alpha\right)^{-1} f(\alpha)
$$

in the space $L_{2}[0, \pi]$, at that,

$$
M f_{n}(\alpha)=(n+1 / 2)^{2} f_{n}(\alpha), \quad \mu_{n}=(n+1 / 2)^{2} .
$$

Thus, in accordance with $(2.2)$, the kernel $G\left(\alpha, \alpha_{0}, z\right)$ of the integral operator

$$
G(z)=(M-z)^{-1}
$$

is represented as

$$
G\left(\alpha, \alpha_{0}, z\right)=\sum_{n=0}^{\infty} \frac{f_{n}(\alpha) f_{n}\left(\alpha_{0}\right)}{\mu_{n}-z}=\sum_{n=0}^{\infty} \frac{\left(n+\frac{1}{2}\right) \sqrt{\sin \alpha \sin \alpha_{0}} P_{n}(\cos \alpha) P_{n}\left(\cos \alpha_{0}\right)}{\left(n+\frac{1}{2}\right)^{2}-z}
$$

Hence, letting

$$
\Gamma\left(\alpha, \alpha_{0}, z\right)=\left(\sin \alpha \sin \alpha_{0}\right)^{-\frac{1}{2}} G\left(\alpha, \alpha_{0}, z\right),
$$

and taking into consideration that $P_{n}(1)=1$, we obtain

$$
\Gamma(\alpha, 0, z)=\sum_{n=0}^{\infty} \frac{(n+1 / 2) P_{n}(\cos \alpha)}{(n+1 / 2)^{2}-z}=\frac{1}{2} \sum_{n=0}^{\infty} \frac{(2 n+1) P_{n}(\cos \alpha)}{n(n+1)-(z-1 / 4)} .
$$

Comparing (2.1) and (2.5), we arrive at the following statement.

Lemma 1. For all $\omega, \omega_{0} \in S^{2}$ and $\lambda \notin\{n(n+1)\}_{n=0}^{\infty}$ the kernel $R_{0}\left(\omega, \omega_{0}, \lambda\right)$ is represented as

$$
R_{0}\left(\omega, \omega_{0}, \lambda\right)=\frac{1}{2 \pi} \Gamma(\alpha, 0, \lambda+1 / 4)
$$

Thus, in view of Lemma 1 and identities (2.3)-(2.6) we see that the kernel $R_{0}\left(\omega, \omega_{0}, \lambda\right)$ can be represented in terms of the solutions to the ordinary differential equations

$$
u^{\prime \prime}+\left(4 \sin ^{2} \alpha\right)^{-1} u+z u=0
$$

in the interval $(0, \pi)$.

We first observe that the identity

$$
\left(4 \sin ^{2} \alpha\right)^{-1}=\left(4 \alpha^{2}\right)^{-1}+q(\alpha)
$$


holds true on the segment $(0, \pi / 2)$, where $q(\alpha) \in C^{(2)}[0, \pi / 2]$. Therefore, we can construct linearly independent solutions to equation (2.7) by means of the solutions to the equation

$$
\nu^{\prime \prime}+\left(4 \alpha^{2}\right)^{-1}+z \nu=0 .
$$

As the linear independent solutions to "unperturbed" equation 2.8 we take the functions (see, for instance, [9, Sect. 1.8])

$$
u_{1}^{0}(\alpha, z)=\sqrt{\alpha} J_{0}(\sqrt{z} \alpha), \quad u_{2}^{0}(\alpha, z)=\sqrt{\alpha} Y_{0}(\sqrt{z} \alpha) \frac{\pi}{2},
$$

where $J_{0}(\sqrt{z} \alpha)$ and $Y_{0}(\sqrt{z} \alpha)$ are the first and second kind Bessel functions, respectively, while the branch of $\sqrt{z}$ is fixed by the restriction $0 \leqslant \arg \sqrt{z}<\pi$.

For the sake of convenience we provide various representations for the cylindric functions, which we will use in what follows.

For the functions $J_{0}(s)$ and $Y_{0}(s)$ we shall employ the expansions ([8, Sect. 5.2]):

$$
\begin{aligned}
& J_{0}(s)=\sum_{k=0}^{\infty} \frac{(-1)^{k}}{(k !)^{2}}\left(\frac{s}{2}\right)^{2 k} \\
& Y_{0}(s)=\frac{2}{\pi} J_{0}(s) \ln \frac{s}{2}-\frac{2}{\pi} \sum_{k=0}^{\infty} \frac{(-1)^{k}}{(k !)^{2}}\left(\frac{s}{2}\right)^{2 k} \psi(k+1) .
\end{aligned}
$$

Here

$$
\psi(k+1)=-\gamma+1+\frac{1}{2}+\ldots+\frac{1}{k}, \quad \psi(1)=-\gamma,
$$

$\gamma$ is the Euler constant. We shall also make use of the asymptotic representations ([8, Sect. 5.11]) for $|s| \gg 1,|\arg s| \leqslant \pi-\delta(\delta>0$ is an arbitrarily small number):

$$
\begin{aligned}
& J_{0}(s)=\sqrt{\frac{2}{\pi s}}\left\{\gamma_{1}(s) \cos \left(s-\frac{\pi}{4}\right)+\gamma_{2}(s) \sin \left(s-\frac{\pi}{4}\right)\right\} \\
& Y_{0}(s)=\sqrt{\frac{2}{\pi s}}\left\{\gamma_{1}(s) \sin \left(s-\frac{\pi}{4}\right)-\gamma_{2}(s) \cos \left(s-\frac{\pi}{4}\right)\right\},
\end{aligned}
$$

where

$$
\gamma_{1}(s)=\sum_{k=0}^{\infty} \frac{(-1)^{k}(0,2 k)}{(2 s)^{2 k}}, \quad \gamma_{2}(s)=\sum_{k=0}^{\infty} \frac{(-1)^{k}(0,2 k+1)}{(2 s)^{2 k+1}},
$$

at that, $(\nu, 0)=1$,

$$
\begin{aligned}
& (\nu, m)=\frac{\left(4 \nu^{2}-1^{2}\right)\left(4 \nu^{2}-3^{2}\right) \cdot \ldots \cdot\left(4 \nu^{2}-(2 m-1)^{2}\right)}{2^{2 m} m !}, \quad m \in \mathbb{N}, \\
& \gamma_{1}(s)=1-\frac{9}{128 s^{2}}+O\left(s^{-4}\right), \quad \gamma_{2}(s)=\frac{1}{8 s}-\frac{75}{1024 s^{3}}+O\left(s^{-5}\right) .
\end{aligned}
$$

It is known (see [9, Sect. 1.8]) that the Wronskian satisfies the identity

$$
W\left(u_{1}^{0}, u_{2}^{0}\right)=u_{1}^{0}(\alpha, z) u_{2}^{0 \prime}(\alpha, z)-u_{1}^{0 \prime}(\alpha, z) u_{2}^{0}(\alpha, z) \equiv 1 .
$$

Now we construct linearly independent solutions to equation 2.7$)$ in the segment $\left(0, \frac{\pi}{2}\right]$ as the solutions to the inhomogeneous Volterra equations

$$
u_{k}(\alpha, z)=u_{k}^{0}(\alpha, z)+\int_{0}^{\alpha} g(\alpha, t, z) q(t) u_{k}(t, z) d t
$$

where

$$
g(\alpha, t, z)=u_{1}^{0}(\alpha, z) u_{2}^{0}(t, z)-u_{1}^{0}(t, z) u_{2}^{0}(\alpha, z)
$$

The solutions $u_{k}(\alpha, z)$ to equation (2.7) constructed on the segment $[0, \pi / 2]$ by means of integral equations 2.15 can be continued to the segment $\left(\frac{\pi}{2}, \pi\right]$, namely, we have the lemma. 
Lemma 2. The solutions to equations (2.7) constructed on the segment $\left[0, \frac{\pi}{2}\right]$ as the solutions to Volterra equations (2.15) can be continued to the segment $\left(\frac{\pi}{2}, \pi\right]$ by the formulae

$$
u_{k}(\alpha, z)=a_{k 1}(z) u_{1}(\pi-\alpha, z)+a_{k 2}(z) u_{2}(\pi-\alpha, z), \quad k=1,2,
$$

where

$$
\left\{\begin{array}{l}
a_{11}(z)=u_{1}\left(\frac{\pi}{2}, z\right) u_{2}^{\prime}\left(\frac{\pi}{2}, z\right)+u_{1}^{\prime}\left(\frac{\pi}{2}, z\right) u_{2}\left(\frac{\pi}{2}, z\right), \quad a_{22}(z)=-a_{11}(z), \\
a_{12}(z)=-2 u_{1}\left(\frac{\pi}{2}, z\right) u_{1}^{\prime}\left(\frac{\pi}{2}, z\right), \quad a_{21}(z)=2 u_{2}\left(\frac{\pi}{2}, z\right) u_{2}^{\prime}\left(\frac{\pi}{2}, z\right) .
\end{array}\right.
$$

Proof. It is easy to see that the functions

$$
\nu_{k}(\alpha, z)=u_{k}(\pi-\alpha, z), \quad \alpha \in\left(\frac{\pi}{2}, \pi\right], \quad k=1,2,
$$

are linearly independent solutions to equation (2.7) on this segment. Then the continuations $u_{k}(\alpha, z)$ are expressed as linear combinations of $\nu_{k}(\alpha, z)$ as $\alpha \in\left(\frac{\pi}{2}, \pi\right]$ :

$$
u_{k}(\alpha, z)=a_{k 1}(z) u_{1}(\pi-\alpha, z)+a_{k_{2}} u_{2}(\pi-\alpha, z),
$$

where $a_{k i}(z)$ are constants depending on $z$ only.

As $\alpha=\pi / 2$, the relations

$$
\left\{\begin{array}{l}
u_{k}\left(\frac{\pi}{2}, z\right)=a_{k 1}(z) u_{1}\left(\frac{\pi}{2}, z\right)+a_{k 2}(z) u_{2}\left(\frac{\pi}{2}, z\right) \\
u_{k}^{\prime}\left(\frac{\pi}{2}, z\right)=-a_{k 1}(z) u_{1}^{\prime}\left(\frac{\pi}{2}, z\right)-a_{k 2}(z) u_{2}^{\prime}\left(\frac{\pi}{2}, z\right)
\end{array}\right.
$$

should hold true.

It is obvious that it follows straightforwardly from relations $2.12-2.14$ that

$$
W\left(u_{1}, u_{2}\right)=W\left(u_{1}^{0}, u_{2}^{0}\right)=1 .
$$

Solving then systems $(2.19)$ and taking into consideration (2.20), we arrive at relations (2.17). The proof is complete.

Now we are in position to formulate the main result of the present section. The following theorem holds true.

Theorem 1. For all $\lambda \in \mathbb{R} \backslash\{n(n+1)\}_{n=0}^{\infty}$ the kernel $R_{0}\left(\omega, \omega_{0}, \lambda\right)$ is represented as

$$
R_{0}\left(\omega, \omega_{0}, \lambda\right)=\frac{1}{2 \pi \sqrt{\sin \alpha}}\left[u_{2}(\alpha, \lambda+1 / 4)-A(\lambda+1 / 4) u_{1}(\alpha, \lambda+1 / 4)\right],
$$

where

$$
A(z)=\frac{1}{2}\left(\frac{u_{2}^{\prime}\left(\frac{\pi}{2}, z\right)}{u_{1}^{\prime}\left(\frac{\pi}{2}, z\right)}+\frac{u_{2}\left(\frac{\pi}{2}, z\right)}{u_{1}\left(\frac{\pi}{2}, z\right)}\right) .
$$

Proof. Let the kernel $D(\alpha, t, z)$ be as follows:

$$
D(\alpha, t, z)= \begin{cases}u_{2}(\alpha, z) u_{1}(t, z), & t \leqslant \alpha \leqslant \pi \\ u_{2}(t, z) u_{1}(\alpha, z), & \alpha \leqslant t \leqslant \pi .\end{cases}
$$

Employing (2.7) and (2.20), we find that

$$
\nu(\alpha, z)=\int_{0}^{\pi} D(\alpha, t, z) h(t) d t
$$

where $h(t) \in L^{2}[0, \pi]$ is a solution of the differential equation

$$
-\nu^{\prime \prime}(\alpha, z)-\left(4 \sin ^{2} \alpha\right)^{-1} \nu(\alpha, z)-z \nu(\alpha, z)=h(\alpha) .
$$

Then the function

$$
u(\alpha, z)=\int_{0}^{\pi} G(\alpha, t, z) h(t) d t
$$


where $G(t, \alpha, z)$ is the kernel of the operator $G(z)$ (see $(2.3)$ ), is represented as

$$
u(\alpha, z)=\int_{0}^{\pi} D(\alpha, t, z) h(t) d t+c u_{1}(\alpha, z),
$$

where we have taken into consideration that $u(\alpha, z)$ should satisfying the finite limit condition:

$$
\lim _{\alpha \rightarrow 0+} u(\alpha, z)(\pi-\alpha)^{-1 / 2} .
$$

As $\alpha<\pi, \alpha \sim \pi$, the right hand of formula 2.25 can be represented as

$$
u(\alpha, z)=u_{2}(\alpha, z) \int_{0}^{\pi} u_{1}(t, z) h(t) d t+c u_{1}(\alpha, z)+W(\alpha, z),
$$

where

$$
W(\alpha, z)=u_{1}(\alpha, z) \int_{\alpha}^{\pi} u_{2}(t, z) h(t) d t-u_{2}(\alpha, z) \int_{\alpha}^{\pi} u_{1}(t, z) h(t) d t,
$$

and it is easy to show that $W(\alpha, z)$ satisfies the estimate

$$
|W(\alpha, z)| \leqslant a_{1}(\pi-\alpha)(1+|\ln (\pi-\alpha)|),
$$

where $a_{1}>0$ is a constant.

Thus, $W(\alpha, z)$ satisfies the condition $\lim _{\alpha \rightarrow \pi-0} \frac{W(\alpha, z)}{\sqrt{\pi-\alpha}}=0$. It implies that the sum of the first two term in the right hand side of (2.27) should satisfy condition (2.26). According to (2.18), this sum can be easily represented as

$$
\begin{aligned}
c\left[a_{11}(z) u_{1}(\pi-\alpha, z)\right. & \left.+a_{12}(z) u_{2}(\pi-\alpha, z)\right] \\
& +\int_{0}^{\pi} u_{1}(t, z) h(t) d t\left[a_{21}(z) u_{1}(\pi-\alpha, z)+a_{22}(z) u_{2}(\pi-\alpha, z)\right] \\
= & {\left[c a_{11}(z)+a_{21}(z) \int_{0}^{\pi} u_{1}(t, z) h(t) d t\right] u_{1}(\pi-\alpha, z) } \\
& +\left[c a_{12}(z)+a_{22}(z) \int_{0}^{\pi} u_{1}(t, z) h(t) d t\right] u_{2}(\pi-\alpha, z) .
\end{aligned}
$$

Since by the definition $u_{1}(\pi-\alpha, z)$ satisfies condition $(2.26)$ and, due to the presence of the logarithmic singularity,

$$
\lim _{\alpha \rightarrow \pi-0} \frac{u_{2}(\pi-\alpha, z)}{\sqrt{\pi-\alpha}}=\infty,
$$

the coefficient at $u_{2}(\pi-\alpha, z)$ should vanish:

$$
c a_{12}(z)+a_{22}(z) \int_{0}^{\pi} u_{1}(t, z) h(t) d t=0 .
$$

Thus, it follows from 2.23, 2.24, 2.25) that

$$
G(\alpha, t, z)=D(\alpha, t, z)-\frac{a_{22}(z)}{a_{12}(z)} u_{1}(\alpha, z) u_{1}(t, z),
$$


where, in accordance with (2.17),

$$
A(z)=\frac{a_{22}(z)}{a_{12}(z)}=\frac{1}{2}\left[\frac{u_{2}^{\prime}\left(\frac{\pi}{2}, z\right)}{u_{1}^{\prime}\left(\frac{\pi}{2}, z\right)}+\frac{u_{2}\left(\frac{\pi}{2}, z\right)}{u_{1}\left(\frac{\pi}{2}, z\right)}\right] .
$$

Then, employing (2.4), (2.5) and the relations

$$
\lim _{\alpha \rightarrow+0} \frac{u_{1}(\alpha, z)}{\sqrt{\alpha}}=\lim _{\alpha \rightarrow+0} \frac{u_{1}^{0}(\alpha, z)}{\sqrt{\alpha}}=1,
$$

we arrive at the statement of the theorem.

\section{The Representation of the Kernel $R_{0 n}\left(\omega, \omega_{0}, \lambda\right)$ OF THE REDUCED RESOlvent}

Representation (2.21) for the kernel $R_{0}\left(\omega, \omega_{0}, \lambda\right)$ allows us to calculate the kernel of the reduced resolvent of the operator $H_{0}$

$$
R_{0 n}\left(\omega, \omega_{0}, \lambda_{n}\right)=\frac{1}{4 \pi} \sum_{m \neq n} \frac{(2 m+1) P_{m}(\cos \alpha)}{m(m+1)-n(n+1)}
$$

in terms of the functions $u_{k}(\alpha, z)$ and their derivatives w.rt. variable $z$

$$
\varphi_{k}(\alpha, z)=\frac{\partial}{\partial z} u_{k}(\alpha, z), \quad \psi_{k}(\alpha, z)=\frac{\partial}{\partial z} \varphi_{k}(\alpha, z), \quad k=1,2,
$$

at the point $\alpha=\frac{\pi}{2}$. Since according to Theorem 1 the spectrum of the operator $H_{0}$ coincides with the poles of the function $A\left(\lambda+\frac{1}{4}\right)$ (for $A(z)$ see formula $(2.22)$ ), that is, with the zeroes of the functions $u_{1}\left(\frac{\pi}{2}, \lambda+\frac{1}{4}\right)$ and $u_{1}^{\prime}\left(\frac{\pi}{2}, \lambda+\frac{1}{4}\right)$ and it is easy to confirm (see [8, Sect. 4.6]) that

$$
\begin{aligned}
& u_{1}\left(\frac{\pi}{2}, \lambda_{n}+\frac{1}{4}\right)=0, \quad n=2 k+1, \quad k=0,1, \ldots, \\
& u_{1}^{\prime}\left(\frac{\pi}{2}, \lambda_{n}+\frac{1}{4}\right)=0, \quad n=2 k, \quad k=0,1, \ldots
\end{aligned}
$$

Assume also that

$$
\varphi_{k}^{0}(\alpha, z)=\frac{\partial}{\partial z} u_{k}^{0}(\alpha, z), \quad \psi_{k}^{0}(\alpha, z)=\frac{\partial}{\partial z} \varphi_{k}^{0}(\alpha, z), \quad k=1,2,
$$

then it follows from (2.15), 2.16) that the functions $\varphi_{k}(\alpha, z)$ and $\psi_{k}(\alpha, z)$ on the segment $\left[0, \frac{\pi}{2}\right]$ are solutions to Volterra equations, namely, the following statement holds.

Lemma 3. For all $k=1,2$ and $z \neq 0$, there exist the derivatives

$$
\begin{aligned}
& \lim _{h \rightarrow 0} \frac{u_{k}(\alpha, z+h)-u_{k}(\alpha, z)}{h}=\varphi_{k}(\alpha, z), \\
& \lim _{h \rightarrow 0} \frac{\varphi_{k}(\alpha, z+h)-\varphi_{k}(\alpha, z)}{h}=\psi_{k}(\alpha, z),
\end{aligned}
$$

in the Banach space $C\left[0, \frac{\pi}{2}\right]$, and $\varphi_{k}(\alpha, z)$ is a solution to the Volterra equation

$$
\varphi_{k}(\alpha, z)=\varphi_{k}^{0}(\alpha, z)+\int_{0}^{\alpha} g_{1}(\alpha, t, z) q(t) u_{k}(t, z) d t+\int_{0}^{\alpha} g(\alpha, t, z) q(t) \varphi_{k}(t, z) d t
$$

where

$$
g_{1}(\alpha, t, z)=\varphi_{1}^{0}(\alpha, z) u_{2}^{0}(t, z)-\varphi_{2}^{0}(\alpha, z) u_{1}^{0}(t, z)+u_{1}^{0}(\alpha, z) \varphi_{2}^{0}(t, z)-u_{2}^{0}(\alpha, z) \varphi_{1}^{0}(t, z)
$$


and

$$
\begin{aligned}
\psi_{k}(\alpha, z)= & \psi_{k}^{0}(\alpha, z)+\int_{0}^{\alpha} g_{2}(\alpha, t, z) q(t) u_{k}(t, z) d t+2 \int_{0}^{\alpha} g_{1}(\alpha, t, z) q(t) \varphi_{k}(t, z) d t \\
& +\int_{0}^{\alpha} g(\alpha, t, z) q(t) \psi_{k}(t, z) d t,
\end{aligned}
$$

where

$$
\begin{aligned}
g_{2}(\alpha, t, z)= & \frac{\partial}{\partial z} g_{1}(\alpha, t, z)=\psi_{1}^{0}(\alpha, z) u_{2}^{0}(t, z)-\psi_{2}^{0}(\alpha, z) u_{1}^{0}(t, z)+u_{1}^{0}(\alpha, z) \psi_{2}^{0}(t, z) \\
& -u_{2}^{0}(\alpha, z) \psi_{1}^{0}(t, z)+2\left(\varphi_{1}^{0}(\alpha, z) \varphi_{2}^{0}(t, z)-\varphi_{2}^{0}(\alpha, z) \varphi_{1}^{0}(t, z)\right) .
\end{aligned}
$$

In what follows, for a representation of the kernel of the reduced resolvent $R_{0 n}\left(\omega, \omega_{0}, \lambda_{n}\right)$ we shall need the following lemma.

Lemma 4. Suppose that functions $f(\lambda)$ and $g(\lambda)$ are twice differentiable in a neighbourhood of $\lambda=\lambda_{n}$ and $g\left(\lambda_{n}\right)=0, g^{\prime}\left(\lambda_{n}\right) \neq 0$. Then

$$
\frac{f(\lambda)}{g(\lambda)}=\frac{f\left(\lambda_{n}\right)}{g^{\prime}\left(\lambda_{n}\right)\left(\lambda-\lambda_{n}\right)}+\left[\frac{f^{\prime}\left(\lambda_{n}\right)}{g^{\prime}\left(\lambda_{n}\right)}-\frac{f\left(\lambda_{n}\right) g^{\prime \prime}\left(\lambda_{n}\right)}{g^{\prime 2}\left(\lambda_{n}\right)}\right]+o(1) .
$$

Proof. In accordance with the assumption of the lemma, the Taylor formula

$$
g(\lambda)=g^{\prime}\left(\lambda_{n}\right)\left(\lambda-\lambda_{n}\right)+\frac{g^{\prime \prime}\left(\lambda_{n}\right)}{2}\left(\lambda-\lambda_{n}\right)^{2}+o\left(\left(\lambda-\lambda_{n}\right)^{2}\right)
$$

holds true. It yields

$$
\begin{aligned}
\frac{1}{g(\lambda)} & =\frac{1}{g^{\prime}\left(\lambda_{n}\right)\left(\lambda-\lambda_{n}\right)}\left[1+\frac{g^{\prime \prime}\left(\lambda_{n}\right)}{2 g^{\prime}\left(\lambda_{n}\right)}\left(\lambda-\lambda_{n}\right)+o\left(\left(\lambda-\lambda_{n}\right)\right)\right]^{-1} \\
& =\frac{1}{g^{\prime}\left(\lambda_{n}\right)\left(\lambda-\lambda_{n}\right)}\left[1-\frac{g^{\prime \prime}\left(\lambda_{n}\right)}{2 g^{\prime}\left(\lambda_{n}\right)}\left(\lambda-\lambda_{n}\right)+o\left(\left(\lambda-\lambda_{n}\right)\right)\right] \\
& =\frac{1}{g^{\prime}\left(\lambda_{n}\right)\left(\lambda-\lambda_{n}\right)}-\frac{g^{\prime \prime}\left(\lambda_{n}\right)}{2 g^{\prime 2}\left(\lambda_{n}\right)}+o(1) .
\end{aligned}
$$

Since the assumptions of the lemma implies that

$$
f(\lambda)=f\left(\lambda_{n}\right)+f^{\prime}\left(\lambda_{n}\right)\left(\lambda-\lambda_{n}\right)+\frac{f^{\prime \prime}\left(\lambda_{n}\right)}{2}\left(\lambda-\lambda_{n}\right)^{2}+o\left(\left(\lambda-\lambda_{n}\right)^{2}\right),
$$

we multiply two latter formulae to obtain the formula for the fraction in the statement of the lemma. The proof is complete.

Since by Theorem 1 the poles of the kernel $R_{0}\left(\omega, \omega_{0}, \lambda\right)$ coincide with the zeroes of the functions $u_{1}\left(\frac{\pi}{2}, z\right), u_{1}^{\prime}\left(\frac{\pi}{2}, z\right)$, where $z=\lambda+\frac{1}{4}$, in view of relations (3.1) and 3.2) we consider separately the cases $n=2 k$ and $n=2 k+1, k=0,1, \ldots$

Let $n=2 k+1$, then denoting

$$
f(\alpha, \lambda)=u_{2}\left(\frac{\pi}{2}, \lambda+\frac{1}{4}\right) u_{1}\left(\alpha, \lambda+\frac{1}{4}\right), \quad g(\lambda)=4 \pi u_{1}\left(\frac{\pi}{2}, \lambda+\frac{1}{4}\right),
$$


in accordance (2.21), (2.22) and by Lemma 4 we have

$$
\begin{aligned}
\frac{f(\alpha, \lambda)}{g(\lambda)}= & -\frac{u_{2}\left(\frac{\pi}{2}, \lambda_{n}+\frac{1}{4}\right) u_{1}\left(\alpha, \lambda_{n}+\frac{1}{4}\right)}{4 \pi \varphi_{1}\left(\frac{\pi}{2}, \lambda_{n}+\frac{1}{4}\right)\left(\lambda-\lambda_{n}\right)} \\
& +\frac{\varphi_{2}\left(\frac{\pi}{2}, \lambda_{n}+\frac{1}{4}\right) u_{1}\left(\alpha, \lambda_{n}+\frac{1}{4}\right)+u_{2}\left(\frac{\pi}{2}, \lambda_{n}+\frac{1}{4}\right) \varphi_{1}\left(\alpha, \lambda_{n}+\frac{1}{4}\right)}{4 \pi \varphi_{1}\left(\frac{\pi}{2}, \lambda_{n}+\frac{1}{4}\right)} \\
& -\frac{\psi_{1}\left(\frac{\pi}{2}, \lambda_{n}+\frac{1}{4}\right) u_{2}\left(\frac{\pi}{2}, \lambda_{n}+\frac{1}{4}\right) u_{1}\left(\alpha, \lambda_{n}+\frac{1}{4}\right)}{8 \pi \varphi_{1}^{2}\left(\frac{\pi}{2}, \lambda_{n}+\frac{1}{4}\right)}+o(1) .
\end{aligned}
$$

Hence, according to relations (2.1), 2.4 , 2.5),

$$
P_{n}\left(\omega, \omega_{0}\right)=\frac{1}{4 \pi} \frac{u_{2}\left(\frac{\pi}{2}, \lambda_{n}+\frac{1}{4}\right) u_{1}\left(\alpha, \lambda_{n}+\frac{1}{4}\right)}{\sqrt{\sin \alpha} \varphi_{1}\left(\frac{\pi}{2}, \lambda_{n}+\frac{1}{4}\right)}=\frac{2 n+1}{4 \pi} P_{n}(\cos \alpha) .
$$

Since

then as $n=2 k+1$

$$
P_{n}(\omega, \omega)=\frac{2 n+1}{4 \pi}, \quad \lim _{\alpha \rightarrow+0} \frac{u_{1}\left(\alpha, \lambda_{n}+\frac{1}{4}\right)}{\sqrt{\alpha}}=1,
$$

$$
\frac{u_{2}\left(\frac{\pi}{2}, \lambda_{n}+\frac{1}{4}\right)}{\varphi_{1}\left(\frac{\pi}{2}, \lambda_{n}+\frac{1}{4}\right)}=2 n+1 .
$$

Remark 1. The case $n=2 k$ can be studied in the same way.

Thus, by Theorem 1, relations (3.6)-(3.8) and Remark 1 we arrive at the following theorem.

Theorem 2. Let $z_{n}=\lambda_{n}+\frac{1}{4}$. The kernels

$$
P_{n}\left(\omega, \omega_{0}\right)=\frac{(2 n+1) P_{n}(\cos \alpha)}{4 \pi} \quad \text { and } \quad R_{0 n}\left(\omega, \omega_{0}, \lambda_{n}\right)
$$

can be represented as

and

$$
P_{n}\left(\omega, \omega_{0}\right)=\frac{(2 n+1) u_{1}\left(\alpha, z_{n}\right)}{4 \pi \sqrt{\sin \alpha}}
$$

$$
R_{0 n}\left(\omega, \omega_{0}, \lambda_{n}\right)=\frac{1}{2 \pi \sqrt{\sin \alpha}}\left[u_{2}\left(\alpha, z_{n}\right)-\frac{2 n+1}{2} \varphi_{1}\left(\alpha, z_{n}\right)-a_{n} u_{1}\left(\alpha, z_{n}\right)\right],
$$

where as $n=2 k+1$,

$$
a_{n}=\frac{u_{2}^{\prime}\left(\frac{\pi}{2}, z_{n}\right)}{\left.2 u_{1}^{\prime}\left(\frac{\pi}{2}, z_{n}\right)\right)}+\frac{\left.(2 n+1) \varphi_{2}\left(\frac{\pi}{2}, z_{n}\right)\right)}{\left.2 u_{2}\left(\frac{\pi}{2}, z_{n}\right)\right)}-\frac{\left.(2 n+1)^{2} \psi_{1}\left(\frac{\pi}{2}, z_{n}\right)\right)}{\left.4 u_{2}\left(\frac{\pi}{2}, z_{n}\right)\right)},
$$

and as $n=2 k$,

$$
a_{n}=\frac{u_{2}\left(\frac{\pi}{2}, z_{n}\right)}{\left.2 u_{1}\left(\frac{\pi}{2}, z_{n}\right)\right)}+\frac{\left.(2 n+1) \varphi_{2}^{\prime}\left(\frac{\pi}{2}, z_{n}\right)\right)}{\left.2 u_{2}^{\prime}\left(\frac{\pi}{2}, z_{n}\right)\right)}-\frac{\left.(2 n+1)^{2} \psi_{1}^{\prime}\left(\frac{\pi}{2}, z_{n}\right)\right)}{\left.4 u_{2}^{\prime}\left(\frac{\pi}{2}, z_{n}\right)\right)} .
$$

While finding the trace formula for a perturbation of the the Laplace-Beltrami operator via the approach of the work [5], the key ingredient is the asymptotics for the second corrector of the perturbation theory

$$
\alpha_{n}=\frac{1}{4 \pi} \int_{0}^{\pi} g(\alpha)(2 n+1) P_{n}(\cos \alpha) R_{0 n}\left(\omega, \omega_{0}, \lambda_{n}\right) \sin \alpha d \alpha ;
$$

for the definition of function $g(\alpha)$ see [5, Formula (7)], at that,

$$
g^{\prime}(0)=g^{\prime}(\pi)=0 \text {. }
$$


Therefore, in view of the representations of the functions $P_{n}\left(\omega, \omega_{0}\right)$ and $R_{0 n}\left(\omega, \omega_{0}, \lambda_{n}\right)$ in Theorem 2, we need to study the asymptotic behavior of the functions $u_{k}(\alpha, z), \varphi_{k}(\alpha, z)$, $\psi_{k}(\alpha, z)$, their derivatives w.r.t. variable $\alpha$, and the numbers $a_{n}$ as $n \rightarrow \infty$. In order to do it, we shall prove a series of statements.

Lemma 5. There exist constants $c_{0}>0$ and $c_{1}>0$ independent of $z$ and $\alpha$ such that

$$
u_{k}(\alpha, z)=u_{k}^{0}(\alpha, z)+\omega_{k}(\alpha, z)
$$

for all $\alpha \in\left[0, \frac{\pi}{2}\right], z>0, k=1,2$. Moreover,

$$
\begin{aligned}
& \left|u_{k}^{0}(\alpha, z)\right| \leqslant c_{0} z^{-1 / 4} \\
& \left|\omega_{k}(\alpha, z)\right| \leqslant c_{1} z^{-3 / 4} \alpha .
\end{aligned}
$$

Proof. Since $\sup _{t \geqslant 0}\left|\sqrt{t} Y_{0}(t)\right|<\infty$ and $\sup _{t \geqslant 0}\left|\sqrt{t} J_{0}(t)\right|<\infty$ (see [9, Sects. 1.71, 7.11]), they imply inequalities $\begin{aligned} & t \geqslant 0 \\ & 3.11\end{aligned}$. Identity 3.10 is implied by 2.15 , where

$$
\omega_{k}(\alpha, z)=\int_{0}^{\alpha} g(\alpha, t, z) q(t) u_{k}(t, z) d t .
$$

Since in accordance with 3.11 and 2.16

$$
|g(\alpha, t, z)|<c_{0}^{2} z^{-1 / 2}
$$

as $z>0$, then the norm of the integral operator in equation 2.15 is bounded from above by the number

$$
2 c_{0}^{2} z^{-1 / 2} \int_{0}^{\frac{\pi}{2}} q(t) d t
$$

Therefore, equation 2.15) yields the estimate

$$
\left\|u_{k}(z)\right\|=\max _{0 \leqslant \alpha \leqslant \frac{\pi}{2}}\left|u_{k}(\alpha, z)\right| \leqslant c_{0}^{2} z^{-1 / 4}+2 c_{0}^{2} z^{-1 / 2} \int_{0}^{\frac{\pi}{2}} q(t) d t\left\|u_{k}(z)\right\| .
$$

It follows that $\sup _{z>0} z^{\frac{1}{4}}\left\|u_{k}(z)\right\|<\infty$. Now estimate 3.12 is implied by $3.13-(3.15$.

We proceed to the studying of the functions

$$
\varphi_{k}^{0}(\alpha, z)=\frac{\partial}{\partial z} u_{k}^{0}(\alpha, z), \quad \psi_{k}^{0}(\alpha, z)=\frac{\partial}{\partial z} \varphi_{k}^{0}(\alpha, z), \quad k=1,2,
$$

and their derivatives w.r.t. the variable $\alpha$. Here we employ the usual notations for the derivatives w.r.t. the variables $\alpha$ :

$$
\begin{aligned}
u_{k}^{0 \prime}(\alpha, z) & =\frac{\partial}{\partial \alpha} u_{k}^{0}(\alpha, z), & u_{k}^{\prime}(\alpha, z) & =\frac{\partial}{\partial \alpha} u_{k}(\alpha, z) \\
\varphi_{k}^{0 \prime}(\alpha, z) & =\frac{\partial}{\partial \alpha} \varphi_{k}^{0}(\alpha, z), & \varphi_{k}^{\prime}(\alpha, z) & =\frac{\partial}{\partial \alpha} \varphi_{k}(\alpha, z) .
\end{aligned}
$$

The following lemma holds.

Lemma 6. For all $\alpha \in\left[0, \frac{\pi}{2}\right], z>0$,

$$
\varphi_{k}^{0}(\alpha, z)=\frac{\alpha}{2 z} u_{k}^{0 \prime}(\alpha, z)-\frac{1}{4 z} u_{k}^{0}(\alpha, z), \quad k=1,2 .
$$


Proof. We have

$$
\varphi_{1}^{0}(\alpha, z)=\frac{\alpha^{3 / 2}}{2 \sqrt{z}} J_{0}^{\prime}(\sqrt{z} \alpha), \quad \varphi_{2}^{0}(\alpha, z)=\frac{\alpha^{3 / 2}}{2 \sqrt{z}} Y_{0}^{\prime}(\sqrt{z} \alpha) .
$$

The derivatives w.r.t. $\alpha$ satisfy

$$
\begin{aligned}
& u_{1}^{0 \prime}(\alpha, z)=\frac{1}{2 \sqrt{\alpha}} J_{0}(\sqrt{z} \alpha)+\sqrt{z \alpha} J_{0}^{\prime}(\sqrt{z} \alpha), \\
& u_{2}^{0 \prime}(\alpha, z)=\frac{1}{2 \sqrt{\alpha}} Y_{0}(\sqrt{z} \alpha)+\sqrt{z \alpha} Y_{0}^{\prime}(\sqrt{z} \alpha),
\end{aligned}
$$

that yields

$$
\begin{aligned}
& J_{0}^{\prime}(\sqrt{z} \alpha)=\frac{1}{\sqrt{z \alpha}} u_{1}^{0 \prime}(\alpha, z)-\frac{1}{2 \sqrt{z} \alpha^{3 / 2}} u_{1}^{0}(\alpha, z), \\
& Y_{0}^{\prime}(\sqrt{z} \alpha)=\frac{1}{\sqrt{z \alpha}} u_{2}^{0 \prime}(\alpha, z)-\frac{1}{2 \sqrt{z} \alpha^{3 / 2}} u_{2}^{0}(\alpha, z) .
\end{aligned}
$$

Now by (3.17), 3.18) and 3.19) we obtain (3.16). The proof is complete.

Lemma 7. For all real $z>0$

$$
\max _{0 \leqslant \alpha \leqslant \frac{\pi}{2}}\left|\varphi_{k}^{0}(\alpha, z)\right| \leqslant c_{0}|z|^{-3 / 4}, \quad k=1,2,
$$

where $c_{0}>0$ is a constant independent of $z$.

Proof. According to 3.11 , the second term in 3.16 can be estimated as $O\left(|z|^{-5 / 4}\right)$ uniformly in $\alpha \in\left[0, \frac{\pi}{2}\right]$, and in accordance with (3.18), (3.19),

$$
\begin{aligned}
& \frac{\alpha}{2} u_{1}^{0 \prime}(\alpha, z)=\frac{1}{2 z} u_{1}^{0}(\alpha, z)+\frac{\alpha}{z^{3 / 4}}\left[\sqrt{\sqrt{z} \alpha} J_{0}^{\prime}(\sqrt{z} \alpha)\right], \\
& \frac{\alpha}{2} u_{2}^{0 \prime}(\alpha, z)=\frac{1}{2 z} u_{2}^{0}(\alpha, z)+\frac{\alpha}{z^{3 / 4}}\left[\sqrt{\sqrt{z} \alpha} Y_{0}^{\prime}(\sqrt{z} \alpha)\right] .
\end{aligned}
$$

By identities (2.10)- 2.13$)$, for the derivatives of the functions $J_{0}(s)$ and $Y_{0}(s)$ we obtain

$$
\begin{aligned}
J_{0}^{\prime}(s) & =\sum_{k=1}^{\infty} \frac{(-1)^{k} k}{(k !)^{2}}\left(\frac{s}{2}\right)^{2 k-1} \\
Y_{0}^{\prime}(s) & =\frac{2}{\pi s} J_{0}(s)+\frac{2}{\pi} J_{0}^{\prime}(s) \ln \frac{s}{2}-\frac{2}{\pi} \sum_{k=1}^{\infty} \frac{(-1)^{k} k}{(k !)^{2}}\left(\frac{s}{2}\right)^{2 k-1} \psi(k+1),
\end{aligned}
$$

while as $s \gg 1$,

$$
\begin{aligned}
J_{0}^{\prime}(s)= & -\frac{1}{2 s} J_{0}(s)-\sqrt{\frac{2}{\pi s}} \sin \left(s-\frac{\pi}{4}\right)\left[\sum_{k=0}^{n}(-1)^{k}(0,2 k)(2 s)^{-2 k}+O\left(s^{-2 n-2}\right)\right] \\
& -\sqrt{\frac{2}{\pi s}} \cos \left(s-\frac{\pi}{4}\right)\left[4 \sum_{k=0}^{n}(-1)^{k}(0,2 k) k(2 s)^{-2 k-3}+O\left(s^{-2 n-3}\right)\right] \\
& -\sqrt{\frac{2}{\pi s}} \cos \left(s-\frac{\pi}{4}\right)\left[\sum_{k=0}^{n}(-1)^{k}(0,2 k+1)(2 s)^{-2 k-1}+O\left(s^{-2 n-3}\right)\right] \\
& +\sqrt{\frac{2}{\pi s}} \sin \left(s-\frac{\pi}{4}\right)\left[2 \sum_{k=0}^{n}(-1)^{k}(0,2 k+1)(2 k+1)(2 s)^{-2 k-2}+O\left(s^{-2 n-4}\right)\right],
\end{aligned}
$$




$$
\begin{aligned}
Y_{0}^{\prime}(s)= & -\frac{1}{2 s} Y_{0}(s)-\sqrt{\frac{2}{\pi s}} \sin \left(s-\frac{\pi}{4}\right)\left\{\left[\sum_{k=0}^{n}(-1)^{k}(0,2 k+1)(2 s)^{-2 k-1}+O\left(s^{-2 n-3}\right)\right]\right. \\
& \left.-\left[4 \sum_{k=0}^{n}(-1)^{k}(0,2 k) k(2 s)^{-2 k-1}+O\left(s^{-2 n-3}\right)\right]\right\} \\
& +\sqrt{\frac{2}{\pi s}} \cos \left(s-\frac{\pi}{4}\right)\left[\sum_{k=0}^{n}(-1)^{k}(0,2 k)(2 s)^{-2 k}+O\left(s^{-2 n-2}\right)\right] \\
& -\sqrt{\frac{2}{\pi s}} \cos \left(s-\frac{\pi}{4}\right)\left[2 \sum_{k=0}^{n}(-1)^{k}(0,2 k+1)(2 k+1)(2 s)^{-2 k-2}+O\left(s^{-2 n-4}\right)\right]
\end{aligned}
$$

Let $N$ be a sufficiently large fixed number. Then by 3.21$),(3.22)$ we find that as $|s| \leqslant N$, the estimates

$$
\left|J_{0}^{\prime}(s)\right| \leqslant c_{1} s, \quad\left|Y_{0}^{\prime}(s)\right| \leqslant \frac{c_{2}}{s}+c_{3} s|\ln s|
$$

hold true, where $c_{k}, k=1,2,3$, are some positive constants.

Then identities (3.16) and estimates (3.25) lead us to the following inequalities:

$$
\begin{aligned}
\left|\varphi_{1}^{0}(\alpha, z)\right| & \leqslant \frac{c_{1} \sqrt{z} \alpha^{5 / 2}}{2 \sqrt{z}} \leqslant \frac{c_{1} N^{5 / 2}}{z^{5 / 4}} \\
\left|\varphi_{2}^{0}(\alpha, z)\right| & \leqslant \frac{c_{2} \alpha^{3 / 2}}{2 \sqrt{z} \sqrt{z} \alpha}+\frac{c_{3}|\ln \sqrt{z} \alpha| \sqrt{z} \alpha \alpha^{3 / 2}}{2 \sqrt{z}} \\
& =\frac{c_{2} \sqrt{\alpha}}{2 z}+\frac{c_{3}(\sqrt{z} \alpha)^{5 / 2}|\ln \sqrt{z} \alpha|}{2 z^{5 / 4}} \leqslant \frac{c_{3} c_{4}}{2 z^{5 / 4}}+\frac{c_{2} \sqrt{\alpha}}{2 z}
\end{aligned}
$$

as $\alpha \sqrt{z} \leqslant N$, where $c_{4}=\max _{0 \leqslant t \leqslant N} t^{5 / 2}|\ln t|$.

If $\alpha \sqrt{z}>N$, in order to estimate $J_{0}^{\prime}(\sqrt{z} \alpha)$ and $Y_{0}^{\prime}(\sqrt{z} \alpha)$, we employ identities (3.23) and (3.24), which imply straightforwardly that as $\alpha \sqrt{z}>N, 3.20$ holds true. The proof is complete.

Lemma 8. As $k=1,2$,

$$
\begin{aligned}
\psi_{k}^{0}(\alpha, z) & =\frac{\partial}{\partial z} \varphi_{k}^{0}(\alpha, z)=-\frac{1}{z} \varphi_{k}^{0}(\alpha, z)-\frac{\alpha^{2}}{4 z} u_{k}^{0}(\alpha, z) \\
& =\left(\frac{1}{4 z^{2}}-\frac{\alpha^{2}}{4 z}\right) u_{k}^{0}(\alpha, z)-\frac{\alpha}{4 z^{2}} u_{k}^{0 \prime}(\alpha, z)
\end{aligned}
$$

for all $\alpha>0$ and $z>0$.

Proof. According to (3.17), we have

$$
\psi_{1}^{0}(\alpha, z)=-\frac{\alpha^{3 / 2}}{4 z^{3 / 2}} J_{0}^{\prime}(\sqrt{z} \alpha)+\frac{\alpha^{5 / 2}}{4 z} J_{0}^{\prime \prime}(\sqrt{z} \alpha) .
$$

Since $J_{0}(s)$ satisfies the equation

$$
J_{0}^{\prime \prime}(s)+\frac{1}{s} J_{0}^{\prime}(s)+J_{0}(s)=0
$$

employing (3.16), we obtain

$$
\begin{aligned}
\psi_{1}^{0}(\alpha, z) & =-\frac{\alpha^{3 / 2}}{4 z^{3 / 2}} J_{0}^{\prime}(\sqrt{z} \alpha)-\frac{\alpha^{5 / 2}}{4 z}\left[\frac{1}{\sqrt{z} \alpha} J_{0}^{\prime}(\sqrt{z} \alpha)+J_{0}(\sqrt{z} \alpha)\right] \\
& =-\frac{\alpha^{3 / 2}}{2 z^{3 / 2}} J_{0}^{\prime}(\sqrt{z} \alpha)-\frac{\alpha^{5 / 2}}{4 z} J_{0}(\sqrt{z} \alpha)=-\frac{1}{4 z} \varphi_{1}^{0}(\alpha, z)-\frac{\alpha^{2}}{4 z} u_{1}^{0}(\alpha, z)
\end{aligned}
$$




$$
=-\frac{\alpha}{2 z^{2}} u_{1}^{0 \prime}(\alpha, z)+\left(\frac{1}{4 z^{2}}-\frac{\alpha^{2}}{4 z}\right) u_{1}^{0}(\alpha, z) .
$$

Similar calculations are true also for $\psi_{2}^{0}(\alpha, z)$. The proof is complete.

Lemma 9. As $k=1,2$,

$$
\varphi_{k}^{0 \prime}(\alpha, z)=\frac{\partial}{\partial \alpha} \varphi_{k}^{0}(\alpha, z)=-\frac{1}{4 z} u_{k}^{0 \prime}(\alpha, z)-\left(\frac{1}{8 \alpha z}+\frac{\alpha}{2}\right) u_{k}^{0}(\alpha, z)
$$

for all $\alpha>0$ and $z>0$.

Proof. We differentiate w.r.t. $\alpha$ in (3.17). Employing (3.16) and (3.27), we obtain

$$
\begin{aligned}
\frac{\partial}{\partial z} \varphi_{1}^{0}(\alpha, z) & =\frac{3 \alpha^{1 / 2}}{4 \sqrt{z}} J_{0}^{\prime}(\sqrt{z} \alpha)+\frac{\alpha^{3 / 2}}{2} J_{0}^{\prime \prime}(\sqrt{z} \alpha) \\
& =\frac{3 \alpha^{1 / 2}}{4 \sqrt{z}} J_{0}^{\prime}(\sqrt{z} \alpha)-\frac{\alpha^{3 / 2}}{2}\left[\frac{1}{\sqrt{z} \alpha} J_{0}^{\prime}(\sqrt{z} \alpha)+J_{0}(\sqrt{z} \alpha)\right] \\
& =\frac{1}{2 \alpha} \varphi_{1}^{0}(\alpha, z)-\frac{\alpha}{2} u_{1}^{0}(\alpha, z)=\frac{u_{1}^{0 \prime}(\alpha, z)}{4 z}-\left(\frac{1}{8 \alpha z}+\frac{\alpha}{2}\right) u_{1}^{0}(\alpha, z) .
\end{aligned}
$$

Similar relations are also true for $\frac{\partial}{\partial \alpha} \varphi_{2}^{0}(\alpha, z)$. The proof is complete.

In the same way, employing (3.26), let us prove the following statement.

Lemma 10. As $k=1,2$,

$$
\psi_{k}^{0 \prime}(\alpha, z)=\frac{\partial}{\partial \alpha} \psi_{k}^{0}(\alpha, z)=\left(\frac{1}{16 \alpha z^{2}}-\frac{\alpha}{4 z}\right) u_{k}^{0}(\alpha, z)-\frac{\alpha^{2}}{4 z} u_{k}^{0 \prime}(\alpha, z)
$$

for all $\alpha>0$ and $z>0$.

Now it is easy to study the asymptotic behavior of the functions $\varphi_{k}(\alpha, z)$ and $\psi_{k}(\alpha, z)$ and of their derivatives by using the above lemmata and equations $(3.3),(3.5)$, respectively.

\section{Estimates NEEDED FOR CALCULATing THE ASYMPtotics OF THE REDUCED RESOLVENT}

Thanks to formulae (2.12), 2.13) and the definition of the functions $u_{k}^{0}(\alpha, z), k=1$, 2 , see (2.9), as $\alpha \sqrt{z}>N \gg 1$ we have

$$
\begin{aligned}
& u_{1}^{0}(\alpha, z)=\sqrt{\frac{2}{\pi}} z^{-1 / 4}\left\{\gamma_{1}(\sqrt{z} \alpha) \cos \left(\sqrt{z} \alpha-\frac{\pi}{4}\right)+\gamma_{2}(\sqrt{z} \alpha) \sin \left(\sqrt{z} \alpha-\frac{\pi}{4}\right)\right\}, \\
& u_{2}^{0}(\alpha, z)=\sqrt{\frac{\pi}{2}} z^{-1 / 4}\left\{\gamma_{1}(\sqrt{z} \alpha) \sin \left(\sqrt{z} \alpha-\frac{\pi}{4}\right)-\gamma_{2}(\sqrt{z} \alpha) \cos \left(\sqrt{z} \alpha-\frac{\pi}{4}\right)\right\}, \\
& \left(u_{1}^{0}\right)^{2}(\alpha, z)=\frac{1}{\pi \sqrt{z}}\left\{\left[\gamma_{1}^{2}(\sqrt{z} \alpha)+\gamma_{2}^{2}(\sqrt{z} \alpha)\right]+\left[\gamma_{1}^{2}-\gamma_{2}^{2}\right] \sin 2(\sqrt{z} \alpha)\right. \\
& \left.-2 \gamma_{1}(\sqrt{z} \alpha) \gamma_{2}(\sqrt{z} \alpha) \cos 2(\sqrt{z} \alpha)\right\} \\
& \left(u_{2}^{0}\right)^{2}(\alpha, z)=\frac{\pi}{4 \sqrt{z}}\left\{\left[\gamma_{1}^{2}(\sqrt{z} \alpha)+\gamma_{2}^{2}(\sqrt{z} \alpha)\right]-\left[\gamma_{1}^{2}-\gamma_{2}^{2}\right] \sin 2(\sqrt{z} \alpha)\right. \\
& +2 \gamma_{1}(\sqrt{z} \alpha) \gamma_{2}(\sqrt{z} \alpha) \cos 2(\sqrt{z} \alpha\} \\
& u_{1}^{0}(\alpha, z) \stackrel{0}{u_{2}}(\alpha, z)=-\frac{1}{2 \sqrt{z}}\left\{\left[\gamma_{1}^{2}(\sqrt{z} \alpha)-\gamma_{2}^{2}(\sqrt{z} \alpha)\right] \cos 2(\sqrt{z} \alpha)\right. \\
& \left.+2 \gamma_{1}(\sqrt{z} \alpha) \gamma_{2}(\sqrt{z} \alpha) \sin 2(\sqrt{z} \alpha)\right\}
\end{aligned}
$$


Here

$$
\gamma_{1}^{2}+\gamma_{2}^{2}=1-\frac{1}{8 t^{2}}+O\left(t^{-4}\right), \quad \gamma_{1}^{2}-\gamma_{2}^{2}=1-\frac{5}{32 t^{2}}+O\left(t^{-4}\right), \quad \gamma_{1} \gamma_{2}=\frac{1}{8 t}+O\left(t^{-3}\right) .
$$

Since we need the values of the functions $u_{k}(\alpha, z)$ and their derivatives at the point $\alpha=\frac{\pi}{2}$, in view of formulae (2.15), 2.16) we shall need the estimates of the functions

$$
\begin{array}{ll}
f_{1}(\alpha, z)=\int_{0}^{\alpha} u_{2}^{0}(t, z) q(t) u_{1}(t, z) d t, \quad f_{2}(\alpha, z)=\int_{0}^{\alpha} u_{1}^{0}(t, z) q(t) u_{1}(t, z) d t, \\
f_{3}(\alpha, z)=\int_{0}^{\alpha} u_{2}^{0}(t, z) q(t) u_{2}(t, z) d t, \quad f_{4}(\alpha, z)=\int_{0}^{\alpha} u_{2}^{0}(t, z) q(t) u_{2}(t, z) d t .
\end{array}
$$

Employing (4.6), 4.7), (3.10), we represent the functions $f_{k}(\alpha, z)$ as

$$
\begin{aligned}
& f_{1}(\alpha, z)=\int_{0}^{\alpha} u_{2}^{0}(t, z) q(t) u_{1}^{0}(t, z) d t+F_{1}(\alpha, z), \\
& F_{1}(\alpha, z)=\int_{0}^{\alpha} u_{2}^{0}(t, z) q(t) w_{1}(t, z) d t, \\
& f_{2}(\alpha, z)=\int_{0}^{\alpha}\left(u_{1}^{0}\right)^{2}(t, z) q(t) d t+F_{2}(\alpha, z), \\
& F_{2}(\alpha, z)=\int_{0}^{\alpha} u_{1}^{0}(t, z) q(t) w_{1}(t, z) d t, \\
& f_{3}(\alpha, z)=\int_{0}^{\alpha} u_{2}^{0}(t, z) q(t) u_{1}^{0}(t, z) d t+F_{3}(\alpha, z), \\
& F_{3}(\alpha, z)=\int_{0}^{\alpha} u_{1}^{2}(t, z) q(t) w_{2}(t, z) d t, \\
& f_{4}(\alpha, z)=\int_{0}^{\alpha}\left(u_{2}^{0}\right)^{2}(t, z) q(t) d t+F_{4}(\alpha, z), \\
& F_{4}(\alpha, z)=\int_{0}^{\alpha} u_{2}^{0}(t, z) q(t) w_{2}(t, z) d t .
\end{aligned}
$$

Lemma 11. For all $\alpha \in[0 ; \pi / 2]$ and $z>0$ the estimate

$$
\left|F_{k}(\alpha, z)\right| \leqslant \frac{c}{z} \alpha^{2}, \quad c>0, \quad k=1,2,3,4,
$$

holds true.

Proof. Indeed, by the definition of the functions $F_{k}$ and estimates (3.11), (3.12) we have

$$
\left|F_{k}(\alpha, z)\right| \leqslant \frac{c}{z} \int_{0}^{\alpha} t d t
$$

that proves the lemma. 
Lemma 12. Let $z_{n} \gg 1$, then

$$
\begin{aligned}
& \int_{0}^{\frac{\pi}{2}} u_{i}^{0}\left(t, z_{n}\right) q(t) u_{j}\left(t, z_{n}\right) d t=O\left(z_{n}^{-1}\right), \quad i \neq j, \quad i, j=1,2, \\
& \int_{0}^{\frac{\pi}{2}} u_{1}^{0}\left(t, z_{n}\right) q(t) u_{1}\left(t, z_{n}\right) d t=\frac{1}{2 \pi^{2} \sqrt{z_{n}}}+O\left(\frac{1}{z_{n}}\right), \\
& \int_{0}^{\frac{\pi}{2}} u_{2}^{0}\left(t, z_{n}\right) q(t) u_{2}\left(t, z_{n}\right) d t=\frac{1}{8 \sqrt{z_{n}}}+O\left(\frac{1}{z_{n}}\right) .
\end{aligned}
$$

Proof. Let $N$ be a sufficiently large fixed number. Since $J_{0}(s) \approx 1, s \rightarrow 0$, as $t \sqrt{z_{n}} \leqslant N$, by expansions (2.10), 2.11) we have

$$
u_{1}^{0}\left(t, z_{n}\right)\left|\leqslant c_{1} \sqrt{t}, \quad\right| u_{2}^{0}\left(t, z_{n}\right)\left|\leqslant c_{2} \frac{t^{\frac{1}{2}-\delta}}{z_{n}^{\frac{\delta}{2}}}\right| u_{1}^{0}\left(t, z_{n}\right) u_{2}^{0}\left(t, z_{n}\right) \mid \leqslant c_{3} \frac{t^{1-\delta}}{z_{n}^{\frac{\delta}{2}}},
$$

where $0<c_{i}, i=1,2,3$, are constants, $\delta$ is a sufficiently small positive number. Therefore,

$$
\left|\int_{0}^{\frac{N}{\sqrt{z_{n}}}}\left(u_{2}^{0}\right)^{2}\left(t, z_{n}\right) q(t) d t\right|<\frac{c}{z^{\delta}} \int_{0}^{\frac{N}{\sqrt{z}}} t^{1-2 \delta} d t=\frac{c}{(2-2 \delta) z^{\delta}}\left(\frac{N}{\sqrt{z_{n}}}\right)^{2-2 \delta}=O\left(\frac{1}{z_{n}}\right) .
$$

In the same way we confirm that

$$
\int_{0}^{\frac{N}{\sqrt{z_{n}}}}\left(u_{1}^{0}\right)^{2}\left(t, z_{n}\right) q(t) d t=O\left(\frac{1}{z_{n}}\right), \quad \int_{0}^{\frac{N}{\sqrt{z_{n}}}} u_{1}^{0}\left(t, z_{n}\right) u_{2}^{0}\left(t, z_{n}\right) q(t) d t=O\left(\frac{1}{z_{n}}\right) .
$$

As $t \sqrt{z_{n}}>N \gg 1$, in order to analyse the integrals

$$
\int_{\frac{N}{\sqrt{z_{n}}}}^{\frac{\pi}{2}} u_{i}^{0}\left(t, z_{n}\right) u_{j}^{0}\left(t, z_{n}\right) q(t) d t
$$

we employ asymptotics formulae (4.1)- 4.5 . Thus,

$$
\begin{aligned}
& \int_{\frac{N}{\sqrt{z_{n}}}}^{\frac{\pi}{2}} u_{1}^{0}\left(t, z_{n}\right) u_{2}^{0}\left(t, z_{n}\right) q(t) d t=-\frac{1}{2 \sqrt{z_{n}}} \int_{\frac{N}{\sqrt{z_{n}}}}^{\frac{\pi}{2}} \cos 2 \sqrt{z_{n}} t\left[1+O\left(\frac{1}{z_{n} t^{2}}\right)\right] q(t) d t \\
& =-\left.\frac{q(t)}{4 z_{n}} \sin 2 \sqrt{z_{n}}\left[1+O\left(\frac{1}{z_{n} t^{2}}\right)\right]\right|_{\frac{N}{\sqrt{z_{n}}}} ^{\frac{\pi}{2}}+\frac{1}{4 z_{n}} \int_{\frac{N}{\sqrt{z_{n}}}}^{\frac{\pi}{2}} O\left(\frac{1}{z_{n} t^{3}}\right) \sin 2 \sqrt{z_{n}} t q(t) d t \\
& +O\left(\frac{1}{z_{n}}\right)=O\left(\frac{1}{z_{n}}\right),
\end{aligned}
$$


since we can choose $N$ so that $\sin 2 N=0$ and

$$
\int_{\frac{N}{\sqrt{z_{n}}}}^{\frac{\pi}{2}} \frac{\sin 2 \sqrt{z_{n}} t}{z_{n} t^{3}} d t=\int_{N}^{\frac{\pi}{2} \sqrt{z_{n}}} \frac{\sin 2 \tau}{\tau^{3}} d \tau
$$

is bounded. In the same way, employing the asymptotic representations for the functions $\left(u_{1}^{0}\right)^{2}\left(t, z_{n}\right)$ and $\left(u_{2}^{0}\right)^{2}\left(t, z_{n}\right)$ from $4.1-4.5$, we obtain:

$$
\begin{aligned}
& \int_{\frac{N}{\sqrt{z_{n}}}}^{\frac{\pi}{2}}\left(u_{1}^{0}\right)^{2}\left(t, z_{n}\right) q(t) d t=\frac{1}{\pi \sqrt{z_{n}}} \int_{\frac{N}{\sqrt{z_{n}}}}^{\frac{\pi}{2}} q(t) d t+O\left(\frac{1}{z_{n}}\right), \\
& \int_{\frac{N}{\sqrt{z_{n}}}}^{\frac{\pi}{2}}\left(u_{2}^{0}\right)^{2}\left(t, z_{n}\right) q(t) d t=\frac{\pi}{4 \sqrt{z_{n}}} \int_{\frac{N}{\sqrt{z_{n}}}}^{\frac{\pi}{2}} q(t) d t+O\left(\frac{1}{z_{n}}\right) .
\end{aligned}
$$

We note that

$$
\int_{0}^{\frac{\pi}{2}} q(t) d t=\frac{1}{2 \pi} .
$$

Now the statement of the lemma is implied by representations (4.6), (4.7), (4.8)-(4.15), Lemma 11 and relations 4.17)-4.22.

\section{ASYMPTOTICS FOR THE SECOND CORRECTOR OF THE PERTURBATION THEORY AND THE TRACE FORMULA}

Basing on Theorem 2 and Lemmata $5 \sqrt{12}$, we proceed to the studying of the asymptotics for the second corrector $\alpha_{n}$ of the perturbation theory, which, as it was mentioned above, is the key point in finding the regularized trace formula for the operator $H$. Namely, the following statement holds.

Theorem 3. Let $g \in W_{2}^{1}[0 ; \pi]$ and $z_{n} \gg 1$. Then the estimate

$$
\alpha_{n}=O\left(z_{n}^{-\frac{3}{4}}\right)=O\left(n^{-\frac{3}{2}}\right)
$$

holds true, i.e., the sequence $\alpha_{n}$ is absolutely summable.

Proof. According to Theorem 2 and (3.9), we have

$$
\begin{aligned}
\alpha_{n} & =\frac{1}{4 \pi} \int_{0}^{\pi}(2 n+1) g(\alpha) P_{n}(\cos \alpha) R_{0 n}\left(\omega, \omega_{0}, \lambda_{n}\right) \sin \alpha d \alpha \\
& =\frac{1}{16 \pi^{2}} \int_{0}^{\pi} g(\alpha) \sqrt{z_{n}} u_{1}\left(\alpha, z_{n}\right)\left[u_{2}\left(\alpha, z_{n}\right)-\sqrt{z_{n}} \varphi_{1}\left(\alpha, z_{n}\right)-a_{n} u_{1}\left(\alpha, z_{n}\right)\right] d \alpha .
\end{aligned}
$$

Since as $\alpha \in\left(\frac{\pi}{2} ; \pi\right)$

$$
u_{k}(\alpha, z)=a_{k 1}(z) u_{1}(\pi-\alpha, z)+a_{k 2}(z) u_{2}(\pi-\alpha, z), k=1,2,
$$

for $\alpha \in\left(\frac{\pi}{2} ; \pi\right)$ we have

$$
\begin{aligned}
\varphi_{1}(\alpha, z)= & a_{11}(z) \varphi_{1}(\pi-\alpha, z)+a_{12}(z) \varphi_{2}(\pi-\alpha, z) \\
& +a_{11}^{\prime}(z) u_{1}(\pi-\alpha, z)+a_{12}^{\prime}(z) u_{2}(\pi-\alpha, z) .
\end{aligned}
$$


Therefore, to study the asymptotic behavior of $\alpha_{n}$ as $n \gg 1$, in view of (5.1) and (5.2), we need to study the asymptotics of the numbers $a_{k i}\left(z_{n}\right), a_{11}^{\prime}\left(z_{n}\right), a_{12}^{\prime}\left(z_{n}\right)$ and $a_{n}$.

Let $n=2 k+1$, i.e., $u_{1}\left(\frac{\pi}{2}, z_{n}\right)=0$ (the case $n=2 k, k=1,2, \ldots$ can be studied in the same way). Since $W\left(u_{1}, u_{2}\right)=1$, we observe that

$$
a_{11}(z)=2 u_{1}\left(\frac{\pi}{2}, z\right) u_{2}^{\prime}\left(\frac{\pi}{2}, z\right)-1
$$

This is why, for $n=2 k+1$ it follows from formulae (2.17), (5.3) that

$$
\begin{aligned}
& a_{11}\left(z_{n}\right)=-1, \quad a_{22}\left(z_{n}\right)=1, \quad a_{12}(z)=-2 u_{1}\left(\frac{\pi}{2}, z_{n}\right) u_{1}^{\prime}\left(\frac{\pi}{2}, z_{n}\right)=0, \\
& a_{21}\left(z_{n}\right)=2 u_{2}\left(\frac{\pi}{2}, z_{n}\right) u_{2}^{\prime}\left(\frac{\pi}{2}, z_{n}\right), \quad a_{12}^{\prime}\left(z_{n}\right)=-2 \varphi_{1}\left(\frac{\pi}{2}, z_{n}\right) u_{1}^{\prime}\left(\frac{\pi}{2}, z_{n}\right), \\
& a_{11}^{\prime}\left(z_{n}\right)=2 \varphi_{1}\left(\frac{\pi}{2}, z_{n}\right) u_{2}^{\prime}\left(\frac{\pi}{2}, z_{n}\right), \\
& \varphi_{1}\left(\alpha, z_{n}\right)=-\varphi_{1}\left(\pi-\alpha, z_{n}\right)+a_{11}^{\prime}\left(z_{n}\right) u_{1}\left(\pi-\alpha, z_{n}\right)+a_{12}^{\prime}\left(z_{n}\right) u_{2}\left(\pi-\alpha, z_{n}\right) .
\end{aligned}
$$

Employing formulae (3.8), 4.1)-4.5) and Lemmata 6 and 8 for $n \gg 1, n=2 k+1$, we obtain

$$
\begin{gathered}
a_{21}\left(z_{n}\right)=O\left(\frac{1}{z_{n}^{\frac{3}{2}}}\right), \quad a_{11}^{\prime}\left(z_{n}\right)=O\left(\frac{1}{z_{n}^{\frac{3}{2}}}\right), \quad a_{12}^{\prime}\left(z_{n}\right)=-\frac{1}{\sqrt{z_{n}}}\left\{1+O\left(\frac{1}{\sqrt{z_{n}}}\right)\right\} . \\
a_{n}=\left[\frac{u_{2}^{\prime}\left(\frac{\pi}{2}, z_{n}\right)}{u_{1}^{\prime}\left(\frac{\pi}{2}, z_{n}\right)}+\frac{z_{n} \varphi_{2}\left(\frac{\pi}{2}, z_{n}\right)}{u_{2}\left(\frac{\pi}{2}, z\right)}-\frac{z_{n}^{2} \psi_{1}\left(\frac{\pi}{2}, z\right)}{u_{2}\left(\frac{\pi}{2}, z\right)}\right]= \\
=\left[O\left(z_{n}^{-1}\right)-\frac{1}{4 \sqrt{z_{n}}}+O\left(z_{n}^{-1}\right)+\frac{1}{2 \sqrt{z_{n}}}+O\left(z_{n}^{-1}\right)\right]=\frac{1}{4 \sqrt{z_{n}}}\left\{1+O\left(\frac{1}{\sqrt{z_{n}}}\right)\right\} .
\end{gathered}
$$

We split the integral in formula (5.1) into that over the segments $\left[0 ; \frac{\pi}{2}\right]$ and $\left[\frac{\pi}{2} ; \pi\right]$. We consider the integral over the second segment and use relations $(5.2)$ and $(\sqrt{5.3)}$, make the change of the variable $\pi-\alpha=t$ and take into consideration identities (5.4)-(5.9) for the numbers $\alpha_{n}$ as $n \gg 1$. As a result, thanks to (5.1), we obtain the following representation:

$$
\begin{aligned}
\alpha_{n}= & \frac{1}{16 \pi^{2}}\left\{\int_{0}^{\frac{\pi}{2}}[g(t)-g(\pi-t)] \sqrt{z_{n}} u_{1}\left(t, z_{n}\right) u_{2}\left(t, z_{n}\right) d t\right. \\
& -\int_{0}^{\frac{\pi}{2}}[g(t)+g(\pi-t)] z_{n} \varphi_{1}\left(t, z_{n}\right) u_{1}\left(t, z_{n}\right) d t \\
& -\int_{0}^{\frac{\pi}{2}}[g(t)+g(\pi-t)] \frac{1}{4 \sqrt{z_{n}}}\left\{1+O\left(\frac{1}{\sqrt{z_{n}}}\right)\right\} u_{1}^{2}\left(t, z_{n}\right) d t \\
& \left.-\int_{0}^{\frac{\pi}{2}} g(\pi-t) \sqrt{z_{n}} u_{1}\left(t, z_{n}\right) u_{2}\left(t, z_{n}\right) d t+O\left(z_{n}^{-\frac{3}{4}}\right)\right\} \\
= & I_{1}(n)+I_{2}(n)+I_{3}(n)+I_{4}(n)+O\left(z_{n}^{-\frac{3}{4}}\right) .
\end{aligned}
$$

Let us study the asymptotic behavior of $I_{j}(n), j=1,2,3,4$, as $n \rightarrow \infty$. We begin with the term $I_{1}(n)$. First we integrate by parts and then, employing estimates (3.11), 3.12) and (4.18) 
and asymptotic representation (4.5), we conclude that

$$
\begin{aligned}
I_{1}(n) & =-\frac{\sqrt{z_{n}}}{16 \pi^{2}} \int_{0}^{\frac{\pi}{2}}\left[g^{\prime}(t)+g^{\prime}(\pi-t)\right] \int_{0}^{t} u_{1}\left(\tau, z_{n}\right) u_{2}\left(\tau, z_{n}\right) d \tau d t \\
& =-\frac{\sqrt{z_{n}}}{16 \pi^{2}}\left\{\int_{0}^{\frac{N}{\sqrt{z n}}} \int_{0}^{t}+\int_{0}^{\frac{N}{\sqrt{z_{n}}}} \int_{\frac{N}{\sqrt{z_{n}}}}^{\frac{\pi}{2}}+\int_{\frac{N}{\sqrt{z_{n}}}}^{\frac{\pi}{2}} \int_{\frac{N}{\sqrt{z_{n}}}}^{t}\right\}\left[g^{\prime}(t)+g^{\prime}(\pi-t)\right] u_{1}\left(\tau, z_{n}\right) u_{2}\left(\tau, z_{n}\right) d \tau d t \\
& =O\left(z_{n}^{-\frac{3}{4}}\right),
\end{aligned}
$$

where $N$ is a sufficiently large fixed number.

In order to study $I_{2}(n)$, we employ representations (2.15), (3.3) and (3.16), estimates (3.11), (3.12) and Lemma 12, as well as asymptotic representations 4.1)-(4.5), to establish that

$$
\begin{aligned}
I_{2}(n)= & -\frac{1}{16 \pi^{2}} \int_{0}^{\frac{\pi}{2}}[g(t)+g(\pi-t)] \frac{t}{2} u_{1}^{0 \prime}\left(t, z_{n}\right) u_{1}\left(t, z_{n}\right) d t \\
& +\frac{1}{64 \pi^{2}} \int_{0}^{\frac{\pi}{2}}[g(t)+g(\pi-t)]\left(u_{1}^{0}\right)^{2}\left(t, z_{n}\right) d t+O\left(z_{n}^{-\frac{3}{4}}\right) \\
= & I_{2}^{(1)}(n)+I_{2}^{(2)}(n)+O\left(z_{n}^{-\frac{3}{4}}\right) .
\end{aligned}
$$

We split the integral $I_{2}^{(1)}(n)$ into two integrals over the segments $\left[0 ; \frac{N}{\sqrt{z_{n}}}\right]$ and $\left[\frac{N}{\sqrt{z_{n}}} ; \frac{\pi}{2}\right]$. Then, using estimates (3.25), (4.16), we get

$$
-\frac{1}{16 \pi^{2}} \int_{0}^{\frac{N}{\sqrt{z_{n}}}}[g(t)+g(\pi-t)] \frac{t}{2} u_{1}^{0 \prime}\left(t, z_{n}\right) u_{1}\left(t, z_{n}\right) d t=O\left(z_{n}^{-\frac{3}{4}}\right) .
$$

For the second segment, employing the asymptotic representation of the function $u_{1}^{0}\left(t, z_{n}\right)$ in (4.1) - 4.5) and integrating once by parts, by the Cauchy-Schwarz inequality and the condition $g \in W_{2}^{1}(0 ; \pi)$ we conclude that

$$
-\frac{1}{16 \pi^{2}} \int_{\frac{N}{\sqrt{z_{n}}}}^{\frac{\pi}{2}}[g(t)+g(\pi-t)] \frac{t}{2} u_{1}^{0 \prime}\left(t, z_{n}\right) u_{1}\left(t, z_{n}\right) d t=\frac{1}{64 \pi^{2} \sqrt{z_{n}}} g\left(\frac{\pi}{2}\right)+O\left(z_{n}^{-\frac{3}{4}}\right) .
$$

Then we observe that

$$
I_{3}(n)+I_{2}^{(2)}(n)=O\left(z_{n}^{-1}\right) .
$$

Finally, let us study the asymptotic behavior of the term $I_{4}(n)$ in formula (5.10). In order to do it, we integrate by parts:

$$
\begin{aligned}
I_{4}(n)= & -\frac{\sqrt{z_{n}}}{16 \pi^{2}} g\left(\frac{\pi}{2}\right) \int_{0}^{\frac{\pi}{2}} u_{1}\left(t, z_{n}\right) u_{2}\left(t, z_{n}\right) d t \\
& -\frac{\sqrt{z_{n}}}{16 \pi^{2}} \int_{0}^{\frac{\pi}{2}} g^{\prime}(\pi-t) \int_{0}^{t} u_{1}\left(\tau, z_{n}\right) u_{2}\left(\tau, z_{n}\right) d \tau d t+O\left(z_{n}^{-1}\right) .
\end{aligned}
$$


Since

$$
\int_{0}^{\frac{\pi}{2}}\left(u_{1}^{0}\right)^{2}\left(t, z_{n}\right) \int_{0}^{t}\left(u_{2}^{0}\right)^{2}\left(\tau, z_{n}\right) q(\tau) d \tau d t-\int_{0}^{\frac{\pi}{2}}\left(u_{2}^{0}\right)^{2}\left(t, z_{n}\right) \int_{0}^{t}\left(u_{1}^{0}\right)^{2}\left(\tau, z_{n}\right) q(\tau) d \tau d t=O\left(z_{n}^{-\frac{3}{2}}\right),
$$

due to Lemmata 5 and 12 we obtain

$$
\int_{0}^{\frac{\pi}{2}} u_{1}\left(t, z_{n}\right) u_{2}\left(t, z_{n}\right) d t=\int_{0}^{\frac{\pi}{2}} u_{1}^{0}\left(t, z_{n}\right) u_{2}^{0}\left(t, z_{n}\right) d t+O\left(z_{n}^{-\frac{3}{2}}\right) .
$$

Employing relations (4.1)-4.5), formula (2) in [10, Sect. 5.12] and the asymptotic representations for the functions $J_{1}(s), Y_{1}(s)$ in [11, Ch. XIII, Sect. A.4] we conclude that

$$
\begin{aligned}
& \int_{0}^{\frac{\pi}{2}} u_{1}^{0}\left(t, z_{n}\right) u_{2}^{0}\left(t, z_{n}\right) d t=\frac{\pi}{2} \int_{0}^{\frac{\pi}{2}} t J_{0}\left(\sqrt{z_{n}} t\right) Y_{0}\left(\sqrt{z_{n}} t\right) d t \\
& =\frac{\pi}{4} u_{1}^{0}\left(\sqrt{z_{n}} \frac{\pi}{2}\right) u_{2}^{0}\left(\sqrt{z_{n}} \frac{\pi}{2}\right)+\frac{\pi^{3}}{16} J_{1}\left(\sqrt{z_{n}} \frac{\pi}{2}\right) Y_{1}\left(\sqrt{z_{n}} \frac{\pi}{2}\right)=\frac{1}{4 z_{n}}+O\left(z_{n}^{-\frac{3}{2}}\right) .
\end{aligned}
$$
that

Thus, according to estimates (5.11) and (5.16), it follows from identities (5.15) and (5.17)

$$
I_{4}(n)=-\frac{1}{64 \pi^{2} \sqrt{z_{n}}} g\left(\frac{\pi}{2}\right)+O\left(z_{n}^{-\frac{3}{4}}\right) .
$$

Now the statement of Theorem 3 is implied by formula (5.10) on the base of identities (5.11), (5.12), 5.13), (5.14) and (5.18).

The proven Theorem 3 allows us to employ the approach of the work [5] for calculating the regularized trace formula and the definition of the function $g(\alpha)$ ( $[5$, Formula (7)]) yields that the smoothness of the functions $g(\alpha)$ and $v(w)$ coincide, we arrive at the main result of the work.

Theorem 4. Let $v(w) \in W_{2}^{1}\left(S^{2}\right)$. Then

$$
\begin{aligned}
\sum_{n=0}^{\infty} \sum_{k=-n}^{n}\left[\mu_{n}^{(k)}-n(n+1)-c_{0}\right]= & \frac{1}{16 \pi^{3}} \int_{S^{2}} \int_{S^{2}} \frac{v(w) v\left(w_{0}\right)}{\sqrt{1-\left(w, w_{0}\right)^{2}}} d \mu(w) d \mu\left(w_{0}\right) \\
& -\frac{1}{8 \pi} \int_{S^{2}} v^{2}(w) d \mu(w),
\end{aligned}
$$

where $\mu_{n}^{(k)}$ are the eigenvalues of the operator $H$,

$$
c_{0}=\frac{1}{4 \pi} \int_{S^{2}} v(w) d \mu(w),
$$

and the series in the left hand side of the above formula converges absolutely.

\section{BIBLIOGRAPHY}

1. V.A. Sadovnichii and V. V. Dubrovskii. A classical regularized trace formula for the eigenvalues of the Laplace-Beltrami operator with a potential on the sphere $S^{2}$ // Dokl. Akad. Nauk SSSR 319:1, 61-62 (1991). [Soviet Math. Dokl. 44:1, 56-58 (1992).]

2. V.E. Podol'skii. The formula of the regularized trace for the Laplace-Beltrami operator with odd potential on the sphere $S^{2}$ // Matem. Zam. 56:1, 71-77 (1994). [Math. Notes. 56:1, 699-703 (1994).] 
3. Z.Yu. Fazullin. Regularized trace formula for the Laplace-Beltrami operator // in "Book of abstracts of International conference on complex analysis and related issues", Nizhni Novgorod, 80-81 (1997). (in Russian).

4. V.A. Sadovnichij, Z.Yu. Fazullin. A formula for the first regularized trace of a perturbed LaplaceBeltrami operator // Diff. Uravn. 37:3, 402-409 (2001). [Diff. Equat. 37:3, 430-438 (2001).]

5. V.A. Sadovnichii, Z.Yu. Fazullin. Asymptotics of the eigenvalues and the formula for the trace of perturbations of the Laplace operator on the sphere $\mathbb{S}^{2} / /$ Matem. Zam. 77:3, 434-448 (2005). [Math. Notes. 77:3, 400-413 (2005).]

6. V.A. Sadovnichii, Z.Yu. Fazullin, A.I. Atnagulov. Properties of the resolvent of the Laplace-Beltrami operator on the two-dimensional sphere and a trace formula // Dokl. AN. 441:2, 174-176 (2011). [Dokl. Math. 84:3, 799-801 (2011).]

7. V.S. Vladimirov. Equations of mathematical physics. Nauka, Moscow (1988). (in Russian).

8. N.N. Lebedev. Special functions and their applications. GIFML, Moscow (1963) [Prentice Hall, Englewood Cliffs, New Jersey (1965).]

9. G. Szegö. Orthogonal polynomials. Amer. Math. Soc, New York. (1959).

10. G.N.Watson. A treatise on the theory of Bessel functions. Cambridge University Press, New York (1944).

11. E. Jahnke, F. Emde, F. Lösch. Tafeln höherer Funktionen. B.G. Teubner Verlagsgesellschaft, Stuttgart (1960) (in German).

Arsen Il'gizovich Atnagulov,

Bashkir State Agrarian Universiy,

50-letie Oktyabrya str. 4,

450080, Ufa, Russia

E-mail: russtudent1@yandex.ru

Sadovnichy Viktor Antonovich,

Lomonosov Moscow State University,

Leninskie gory, 1 ,

119991, Moscow, Russia

E-mail: rector@rector.msu.su

Ziganur Yusupovich Fazullin,

Bashkir State University,

Zaki Validi str. 32,

450074, Ufa, Russia

E-mail: fazullinzu@mail.ru 